\title{
Assessment of the effect of blasting vibaration on tunnel lining of Hai Van Pass Tunnel during expanding excavation the auxiliary tunnel by drilling and blasting method
}

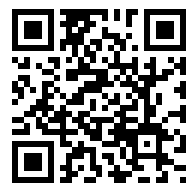

\section{Kien Van Dang *}

Faculty of Civil Engineering, Hanoi University of Mining and Geology, Viet Nam

\begin{tabular}{|c|c|}
\hline ARTICLE INFO & ABSTRACT \\
\hline $\begin{array}{l}\text { Article history: } \\
\text { Received 28th Aug. } 2020 \\
\text { Revised 7th Nov. } 2020 \\
\text { Accepted 31st Dec. } 2020\end{array}$ & $\begin{array}{l}\text { Experimental and numerical research of the effect of blasting vibrations } \\
\text { during tunneling on surrounding structures (rock mass, existing tunnels, } \\
\text { buildings, etc.) was widely studied in recent years. However, the effect of } \\
\text { blasting vibration from a new tunnel on an existing adjacent tunnel is still }\end{array}$ \\
\hline $\begin{array}{l}\text { Keywords: } \\
\text { Blasting vibrations, } \\
\text { Rock mass, } \\
\text { Surrounding structures, } \\
\text { Tunneling. }\end{array}$ & $\begin{array}{l}\text { unclear.A few researches has been carried out to study the relationship of } \\
\text { the observed Peak Particle Velocity (PPV) on the lining areas along the } \\
\text { existing tunnel direction, due to either the lack of in situ test data or the } \\
\text { difficulty in conducting field tests, particularly for tunnels that are usually } \\
\text { old and vulnerable after several decades of service. The paper uses the } \\
\text { synthetic analysis method, study result of Croix-Rousse, Lyon, France to } \\
\text { propose on assessing the effects of blasting vibration on the existing lining } \\
\text { of the main tunnel during expanding excavation the auxiliary tunnel by } \\
\text { drilling and blasting method in similar conditions at Hai Van Pass Tunnel } \\
\text { in Viet Nam. }\end{array}$ \\
\hline
\end{tabular}

${ }^{*}$ Corresponding author

E - mail: dangvankien@humg.edu.vn

DOI: $10.46326 /$ JMES.HTCS2020.18 


\title{
Đánh giá chấn động nổ mìn đến kết cấu vỏ chống của đường hầm Hải Vân khi tiến hành đào mở rộng hầm lánh nạn bằng phương pháp khoan nổ mìn
}

\author{
Đặng Văn Kiên* \\ Khoa Xây dựng, Trưòng Đại học Mỏ - Địa chất, Vietnam
}

THÔNGTINBÀI BÁO

Quá trình:

Nhận bài 28/8/ 2020

Sửa xong $7 / 11 / 2020$

Chấp nhận đăng 31/12/ 2020

Từ khóa:

Chấn động nổ mìn,

Công trình lân cận,

Đào hầm,

Khối đá.

\section{TÓMTẮT}

Nghiên cứu thực nghiệm và mô hình số về ảnh hưởng của chấn động nổ mìn trong quá trình đào hầm lên các công trình lân cận (khối đá, đường hầm tồn tại trước đó, các tòa nhà, v.v.) đã được nghiên cứu rộng rãi trong nhũng năm gần đây. Tuy vậy, ảnh hưởng của chấn động nổ mìn từ đường hầm mới đối với đường hầm lân cộn hiện tại vẫn chưa nghiên cúu đầy đủ. Một số nghiên cứu đã được thực hiện để nghiên cúu mối quan hệ của vận tốc dao động đỉnh của phần từ (Peak Particle Velocity) trong vỏ chống bê tông của đường hầm cũ doc theo truc đường hầm. Tuy nhiên, do thiếu dũ liệu thử nghiệm hiên trường hoặc khó khăn trong việc tiến hành thử nghiệm hiện trường, và việc sử lý kết quả đo, đặc biệt với đường hầm cũ tồn tại lâu bị hu hỏng sau một thời gian dài sử dụng. Bài báo sư dụng phương pháp phân tích tổng hợp sử dụng các kết quả nghiên cúu của hầm Croix-Rousse, Lyon, Pháp để đề xuất đánh giá ảnh hưởng của chấn động nổ mìn đến vỏ bê tông liền khối của hầm chính trong quá trinh đào mở rộng hầm phụ bằng trong điều kiện tương tự tại hầm đèo Hải Vân ở Việt Nam.

(C) 2020 Trường Đại học Mỏ - Địa chất. Tất cả các quyền được bảo đảm.

\section{Mở đầu}

Hầm đường bộ Hải Vân là một trong 30 dự án hầm lớn và hiện đại nhất thế giới, trong đó có hai hầm: hầm chính (dài $6.345 \mathrm{~m}$, chiều rộng xe chạy $10 \mathrm{~m}$, diện tích mặt cắt ngang hầm $80 \mathrm{~m}^{2}, 2$ làn xe) và hầm tránh hay hầm lánh nạn chiều dài 6.359 m,

\footnotetext{
*Tác giả liên hệ

E - mail: dangvankien@ humg.edu.vn DOI: 10.46326/ JMES.HTCS2020.18
}

chiều rộng hầm $4,7 \mathrm{~m}$, diện tích mặt cắt ngang phần thân hầm là $15,46 \mathrm{~m}^{2}$, phần cửa hầm là 22,2 $\mathrm{m}^{2}$, không có xe qua lại). Khoảng cách giữa hai hầm không lớn là $30 \mathrm{~m}$ như Hình 1 (Ichizuru Ishimoto, 2004). Sau hơn 10 năm sử dụng và khai thác (từ năm 2005) hầm đường bộ Hải Vân đã bộc lộ nhiều nhược điểm với thiết kế trên như: lưu lượng xe thường xuyên quá tải, nhất là vào những lúc cao điểm, dẫn đến mất an toàn giao thông, gây raùn tắc. Ngoài ra, hầm đã xảy ra các vụ cháy trong đường hầm do hạn chế của hệ thống thông gió khi sử dụng một đường hầm chính với hai chiều,... Vi 
vậy, việc đầu tư mở rộng hầm lánh nạn thành hầm giao thông của dự án Hâm đường bộ Hải Vân nhằm đáp ứng nhu cầu giao thông vận tải hiện tại, giảm thiếu các rủi do mất an toàn trong khi vận hành là vô cùng cần thiết. Việc thi công mở rộng hầm lánh nạn gặp nhiều khó khăn như phần đất đá xung quanh hầm được gia cố vững chắc bằng các kết cấu chống theo phương pháp đào hầm mới của Áo (NATM), khoảng cách giữa hầm lánh nạn và hầm chính quá gần,... Do vậy, việc nghiên cứu dự báo chấn động nổ mìn nhằm điều chỉnh khối lượng thuốc nổ lớn nhất cho một đợt nổ, lượng thuốc lớn nhất cho một hộ chiếu nhằm đảm bảo an toàn cho vỏ chống bê tồng cố định của đường hầm chính là hết sức quan trọng.

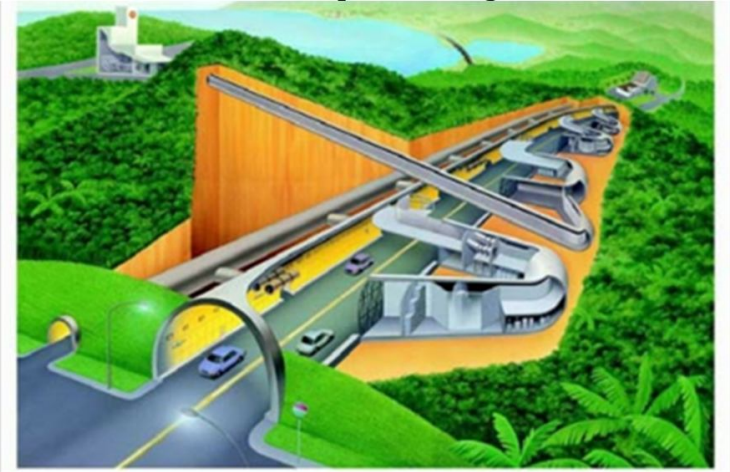

a) Phôi cảnh dự án hầm đường bộ Hải Vân (Ichizuru Ishimoto, 2004)

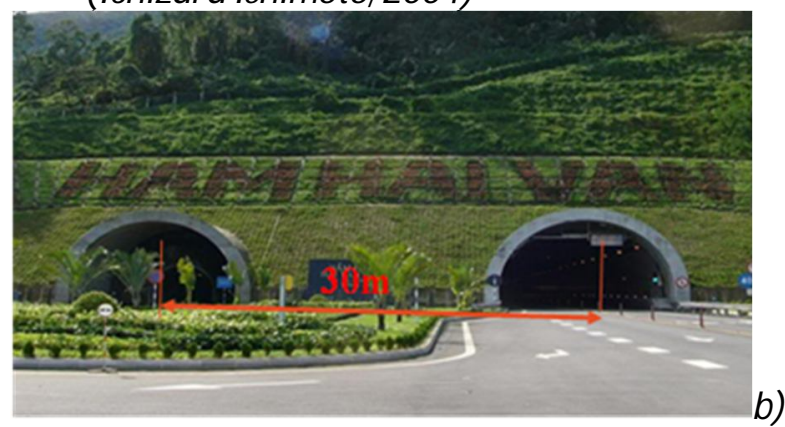

b) Khoảng cách hai đường hầm

Hình 1. Dư án hầm Hải vân.

\section{Tổng quan về các vấn đề kỹ thuật khi mở rộng dự án hầm Hải Vân}

\section{1. Đặc điểm vỏ chống bê tông cố định hầm chính của dự án hầm Hải Vân}

Tại dự án hầm Hải Vân, kết cấu vỏ chống bê tông cố định của đường hầm chính và hầm lánh nạn thay đổi theo từng loại đường hầm và điều kiện địa chất khu vực hầm đào qua. Hâm chính Hải Vần được thi công theo NATM, vỏ chống gồm hai lớp chính: lớp vỏ chống tam theo NATM thay đổi theo từng loại đá với chiều dày $0,05 \div 0,25 \mathrm{~m}$, lớp vỏ chống cố định bên trong là bê tông xi măng Portland đúc tại chỗ dày $30 \mathrm{~cm}$, loại B. Hâm lánh nạn có lớp vỏ chống cố định là bê tông xi măng Porland dày $20 \mathrm{~cm}$.

\section{2. Đặc tính các loại đá hầm đào qua}

Hầm Hải Vân được thi công theo phương pháp NATM, đất đá xung quanh đường hầm và trên gương được chia làm 6 loại. Theo tài liệu khảo sát địa chất đường hầm chủ yếu đào qua đá granit thuộc loại I và loại II như sau:

Loại đá I: Loại đá này gồm các lớp đá cứng, nứt nẻ nhẹ nhưng ổn định. Do đá nứt nên có thể có những mảnh đá rơi nhưng những mảnh này không gây tác hại cho tính ổn định của gương đào. Với loại đá này sự biến dạng ban đầu có tính chất đàn hồi là không đáng kể.

Loại đá II: Loại đá này gồm có đá cứng, nứt nẻ nhẹ nhưng ổn định. Do các vết nứt trong đá, những mảnh đá này có thể rơi ra, tuy nhiên tính ổn định của gương đào vẫn có thể duy trì được: đá có thể chịu đựng được vài ngày. Với loại đá này sự biến dạng ban đầu là rất nhỏ.

Trên cơ sở đó, bài báo sẽ tập trung nghiên cứu chủ yếu đá loại I và loại II là đán rắn cứng có điều kiện giống với đá tại dự án hầm Croix-Rousse, Lyon, Pháp. Việc thi công đường hầm bằng phương pháp khoan nổ mìn thông qua việc dùng một lượng thuốc nổ lớn để phá võ do vậy sẽ gây ảnh hưởng đến vỏ chống bê tông của hầm chính.

\subsection{Phương pháp đào mở rộng hầm Hải Vân}

Do hầm lánh nạn đào trong khối đá rắn cứng (gồm đá granite và một số loại đá khác có độ bền cao) nên phương pháp đào mở đang sử dụng là phương pháp khoan nổ mìn vi sai tạo biên do hiệu quả thi công cao và giá thành hợp lý. Phương pháp nổ trên cho phép giảm chấn động đến khối đá xung quanh đường hầm, đồng thời giảm chi phí về kết cấu chống giữ đường hầm nhằm sử dụng tối đa sự mang tải của khối đá xung quanh, giảm chấn động đến vỏ chống bê tông cố định của đường hầm chính ở khoảng cách gần. Mặc dù vậy, phương pháp vẫn có nhược điểm là gây chấn động đến khối đá và hình thành các khe nứt thứ sinh, ngoài ra trong quá trình nổ mìn đào hầm chấn động gây ra bởi công tác nổ mìn sẽ ảnh hưởng lớn 
đến vỏ chống bê tông của đường hầm chính nằm cách đó khoảng 30 m như Hình 1. Hộ chiếu khoan nổ mìn mở rộng điển hình hầm lánh nạn được thể hiện trên Hình 2.

Một trong các giải pháp giảm chấn động đến vỏ chống bê tông cố định của đường hầm chính là khống chế khối lượng thuốc nổ đồng thời lớn nhất dựa trên vận tốc dao động đỉnh phần tử cho phép và khoảng cách từ vị trí gương hầm mở rộng đến vỏ chống bê tông của đường hầm dựa trên các số liệu đo đạc tại hiện trường trên cơ sở các loại đá khác nhau. Theo định nghĩa, khối lượng thuốc nổ lớn nhất đồng thời là khối lượng thuốc nổ nổ tức thời lớn nhất trong khoảng thời gian $8 \mathrm{~ms}$ (Olsson, M, 2001; Saharan và n.n.k, 2008).

\subsection{Phương pháp nghiên cứu dự báo chấn động nổ mìn hầm Hải Vân}

Hiện nay trên thế giới khi nghiên cứu ảnh hưởng của chấn động nổ mìn người ta thường sử dụng hai phương pháp chủ yếu gồm:

Phương pháp đo đạc hiện trường, sử dụng các Cảm biến đo vận tốc dao động đỉnh phần tử (Peak Particle Velocity-PPV); đo gia tốc dao động đỉnh phần tử (Peak Particle Acceleration-PPA) và chuyển vị của các phần tử (Displacement,...) theo các dải tần số khác nhau. Trong các nghiên cứu (Dang V.K, 2013; Đặng Văn Kiên, 2014; Đặng Văn Kiên; 2016, Đặng Văn Kiên, 2017; F. L. Pellet, V. K. Dang và n.n.k, 2013); (M. Keshavarz, V.K. Dang và n.n.k, 2013); (Van Kien DANG, 2013; Van Kien Dang, 2016; Võ Trọng Hùng và n.n.k, 2018). tác giả sử dụng dữ liệu đo đạc tại hầm Croix-Rousse, Lyon, CH Pháp là kết quả của phương pháp đo đạc thực nghiệm để nghiên cứu đặc tính truyền sóng, kiểm chứng mô hình số xây dựng được. Từ kết quả đo đạc sẽ tiến hành phân tích dữ liệu đo, xây dựng các quy luật về sự ảnh hưởng của các thông số cơ lý của khối đá, đặc tính và khối lượng thuốc nổ lớn nhất đồng thời, khoảng cách từ vị trí nổ mìn đến vị trí quan sát từ đó xây dựng các công thức kinh nghiệm cho phép xác định khối lượng thuốc nổ lớn nhất đồng thời, khối lượng thuốc nổ cho một chu kỳ nổ nhằm đảm bảo an toàn cho vỏ chống bê tông của đường hầm cũ (Dang V.K, 2013; Đặng Văn Kiên, 2014; Đặng Văn Kiên; 2016, Đặng Văn Kiên, 2017; F. L. Pellet, V. K. Dang và n.n.k, 2013); (M. Keshavarz, V.K. Dang và n.n.k, 2013); (Van Kien DANG, 2013; Van Kien Dang, 2016; Võ Trọng Hùng và n.n.k, 2018).
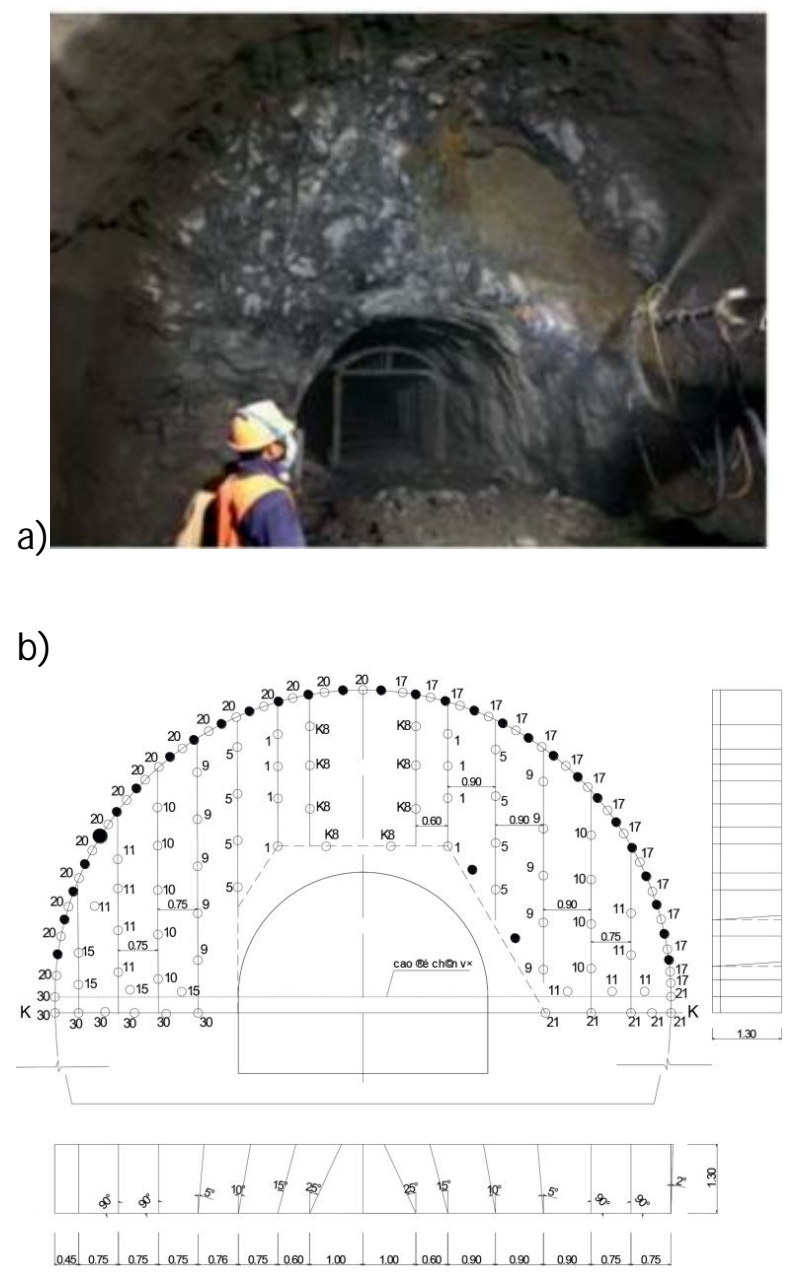

Hình 2. Công tác khoan gương mở rộng hầm lánh nạn (a) và hộ chiếu khoan nổ mìn mở rộng điển hình hầm lánh nạn (b).

Phương pháp mô hình số được xây dựng trên phương pháp phần tử hữu hạn, sai phân hữu hạn, phần tử biên,... Các mô hình số được xây dựng trong điều kiện gần giống với điều kiện thực tế cho phép dự báo tương đối chính xác chấn động gây ra bởi nổ mìn đào hầm . Sự kết hợp của hai phương pháp thực nghiệm và phương pháp số bằng phần mềm Abaqus sẽ đưa ra kết quả dự báo tương đối chính xác ảnh hưởng của chấn động nổ mìn đến kết cấu chống công trình ngầm. Thông qua kết quả đo đạc, các kết quả của mô hình số được kiểm chứng và được sử dụng dự báo trong các điều kiện tương tự. Ngoài ra, dựa trên mô hình số đã được kiểm chứng chúng ta có thể tiến hành khảo sátảnh hưởng của các thông số đầu vào như: đặc tính cơ lý của đất đá, đặc tính áp lực nổ của thuốc nổ, hệ số giảm chấn đến chấn động nổ mìn. Mặc dù khi tiến hành mở rộng hầm lánh nạn tại dự án hầm 
đường bộ qua đề Hải Vân có sử dụng phương pháp đo đạc hiện trường tuy nhiên qua đánh giá cho thấy các dũ liệu đo chưa hoàn toàn được kiểm chứng và khó sử dụng để kết hợp với mô hình số. Bài báo sử dụng kết quả của một số nghiên cứu trước đã được công bố cho hầm Croix-Rousse, Lyon, Pháp để áp dụng cho việc dự báo chấn động nổ mìn cho hầm Hải Vân trong điều kiện tương tự khi hầm đi qua đá granite rắn cứng cần chi phí một lượng thuốc nổ lớn.

\subsection{Một số thông số đánh giá ảnh hưởng của chấn động nổ mìn theo các tiêu chuẩn của Việt Nam}

Khi đánh giá ảnh hưởng của chấn động nổ mìn đến khối đá hoặc các công trình lân cận người ta cần sử dụng các thông số cơ bản cho phép có thể đánh giá được ảnh hưởng của chấn động nổ mìn và là cơ sở để tiến hành đo chấn động tại hiện trường từ đó điều chỉnh lượng thuốc nổ lớn nhất cho mỗi lần nổ mìn nhằm giảm thiểu chấn động đến vỏ chống bê tông, hạn chế các vết nứt xuất hiện trong vỏ khi tiến hành nổ mìn đào hầm.

Các thông số đánh giá ảnh hưởng của chấn động nổ mìn. Để đánh giá chấn động nổ mìn khi đào hầm bằng phương pháp khoan nổ mìn người ta thường sử dụng các thông số cơ bản bao gồm:

Vận tốc dao động phần tử lớn nhất- PPV;

Gia tốc dao động phần tử lớn nhất;

Chuyển vị phần tử lớn nhất;

Tần số dao động riêng của kết cấu công trình.

Trong các thông số trên thì hai thông số vận tốc dao động lớn nhất và tần số dao động riêng của công trình được sử dụng nhiều hơn cả. Trong nghiên cứu này kiến nghị sử dụng hai thông số là vận tốc dao động phần tử lớn nhất và tần số dao động để nghiên cứu đánh giá chấn động tại dự án hầm Hải Vân.

\subsection{Tiêu chuẩn quy phạm của Việt nam về đánh giá ảnh hướng của chấn động nổ mìn}

Tại Việt nam hiện nay đang sử dụng Quy phạm QCVN 02_2008_BCT của Bộ công thương, tỉ lệ khoảng cách và giá trị vận tốc dao động phần tử lớn nhất để đảm bảo công trình an toàn phải thỏa mãn yêu cầu trong Bảng 1 (QCVN 02:2008/ BCT). Ngoài ra cho phép áp dụng đồ thị Hình 3 để xác định mức cho phép của vận tốc dao động phần tử cực trị ở dải tần số thấp thay cho Bảng 1 .
Bảng 1. Hệ số tỉ lệ khoảng cách và vận tốc dao động lón nhất theo quy phạm (QCVN 02:2008/BCT).

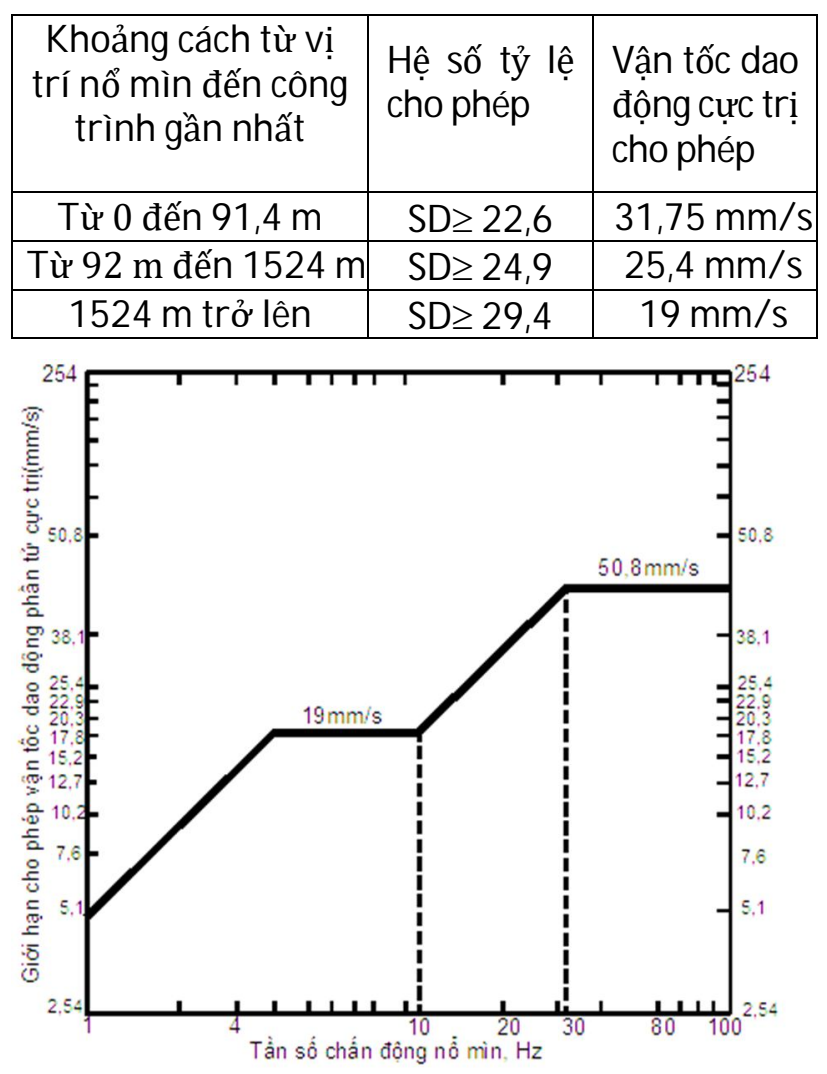

Hình 3. Mức cho phép của vận tốc dao động phần tứ cực trị ở dải tần số thấp (QCVN 02:2008/ BCT).

Tại Việt Nam, nhà thầu khi thi công các dự án như hầm đường sắt Uông Bí hầm đường bộ qua đèo Cả (Tổng công tu Sông Đà), dự án mở rộng hầm Hải Vân (Công ty CP Đèo Cả) không có thiết bị đo chuyên dụng nên khi phải giám sát chấn động thường thuê một đơn vị thứ ba. Hiện tại ở Việt Nam, Hội Kỹ thuật nổ mìn đang có thiết bị này.

Tại hầm Hải Vân và Cổ Mã, Hội đã tiến hành sử dụng thiết bị đo Minimate Plus của hãng Instantel để tiến hành đo chấn động hầm đường sắt nhằm điều chỉnh lượng thuốc nổ lớn nhất cho mỗi lần nổ mìn nhằm giảm thiểu chấn động đến vỏ chống bê tông, hạn chế các vết nứt xuất hiện trong vỏ khi tiến hành nổ mìn đào hầm. Tại đây cũng đã sử dụng Quy phạm QCVN 02 2008 BCT của Bộ công thương để đánh giá chấn động cho vỏ chống của hầm đường sắt và kết quả là vỏ chống an toàn. Tuy nhiên việc đánh giá chấn động tại dự án này mới dừng lại ở việc đo đạc và so sánh kết quả đo với các tiêu chuẩn quy phạm hiện hành của Việt Nam. Với nhược điểm là chỉ có kết quả đo mới có thể 
đánh giá được chấn động trong khi việc đo đạc thường xuyên là rất khó nên việc phát triển các mô hình số để đánh giá chấn động trong các điều kiện tương đương là hết sức cần thiết.

\section{3. Đánh giá chấn động nổ mìn đến kết cấu vỏ chống của đường hầm Hải Vân khi tiến hành đào mở rộng hầm lánh nạn bằng phương pháp khoan nổ mìn}

Bài báo sử dụng kết quả nghiên cứu của tác giả trong các công trình (Dang V.K, 2013; Đặng Văn Kiên, 2014; Đặng Văn Kiên; 2016, Đặng Văn Kiên, 2017; F. L. Pellet, V. K. Dang và n.n.k, 2013); (M. Keshavarz, V.K. Dang và n.n.k, 2013) ; ( Van Kien DANG, 2013; Van Kien Dang, 2016; Võ Trọng Hùng, Đặng Văn Kiên và n.n.k, 2018) về đánh giá ảnh hưởng của chấn động nổ mìn khi nổ mìn hầm mới đến vỏ chống hầm cũ tại dự án hầm CroixRousse, Lyon, Pháp. Trên cơ sở kết quả các dữ liệu đo đạc từ thực tế và các kết quả đạt được từ quá trình khảo sát các mô hình số trên đây, tác giả đã tiến hành xây dựng mối tương quan giữa giá trị PPV và khoảng cách tính từ gương đường hầm đến các điểm khảo sát $(\mathrm{D})$ dọc theo trục đường hầm chính của dự án hầm đường bộ qua Đèo Hải Vân cho hai trường hợp các điểm quan sát trước gương hầm lánh nạn $(\mathrm{H}>0)$ và các điểm trong vỏ chống bê tông của hầm chính sau gương hầm lánh nạn $(\mathrm{H}<0)$ như sơ đồ Hình 4 . Trên cơ sở đó, tác giả tiến hành xây dựng các công thức thực nghiệm dự báo giá trị PPV trong vỏ chống bê tông của đường hầm hầm chính trong khối đá granite với điều kiện như của hầm Croix-Rousse, Lyon, Pháp.

Áp dụng kết quả nghiên cứu cho đường hầm đi qua khu vực đất đá granite với điều kiện tương tự như hầm Croix-Rousse. Khi điều chỉnh khoảng cách từ gương hầm mở rộng tại hầm lánh nạn đến vị trí điểm khảo sát trong vỏ chống hầm chính (D) ta nhận được biểu đồ mối quan hệ giữa vận tốc dao động phần tử lớn nhất với khoảng cách (D) giữa gương hầm lánh nạn và điểm khảo sát trong vỏ bê tông dọ trục hầm chính cho hai trường hợp $\mathrm{H}>0$ và $\mathrm{H}<0$ như Hình 5 .

Từ biểu đồ Hình 5 ta vẽ đường gần đúng (đường màu đen), trên cơ sở đó xác định được phương trình gần đúng của hàm PPV theo khoảng cách (D) cho phép xác định giá trị PPV trong vỏ chống bê tông của đường hầm chính cho các điểm phía trước gương hầm có thể dự báo gần đúng bằng công thức (1), ngược lại các điểm phía sau gương hầm trong môi trường khối đá granite có thể dự báo gần đúng bằng công thức (2). $0)$ :

$\mathrm{PPV}=\left(-0,0002 \mathrm{D}^{3}+0,0237 \mathrm{D}^{2}\right.$

$0,9252 \mathrm{D}+15,4487) ; \quad \mathrm{R}^{2}=0,9991$

$>$ Các điểm phía sau gương đường hầm $(\mathrm{H}>0)$ : $\mathrm{PPV}=\left(0,00035 \mathrm{D}^{3}+0,03349 \mathrm{D}^{2}-\right.$

$1,08508 \mathrm{D}+15,20692) ; \mathrm{R}^{2}=0,9955$

Việc sử dụng các công thức (1) và (2) có thể cho phép dự báo giá trị của PPV xuất hiện trong khối đá granite trong điều kiện thi công mở rộng dự án hầm Hải Vân nhằm đảm bảo an toàn cho vỏ chống bê tông của đường hầm lánh nạn.

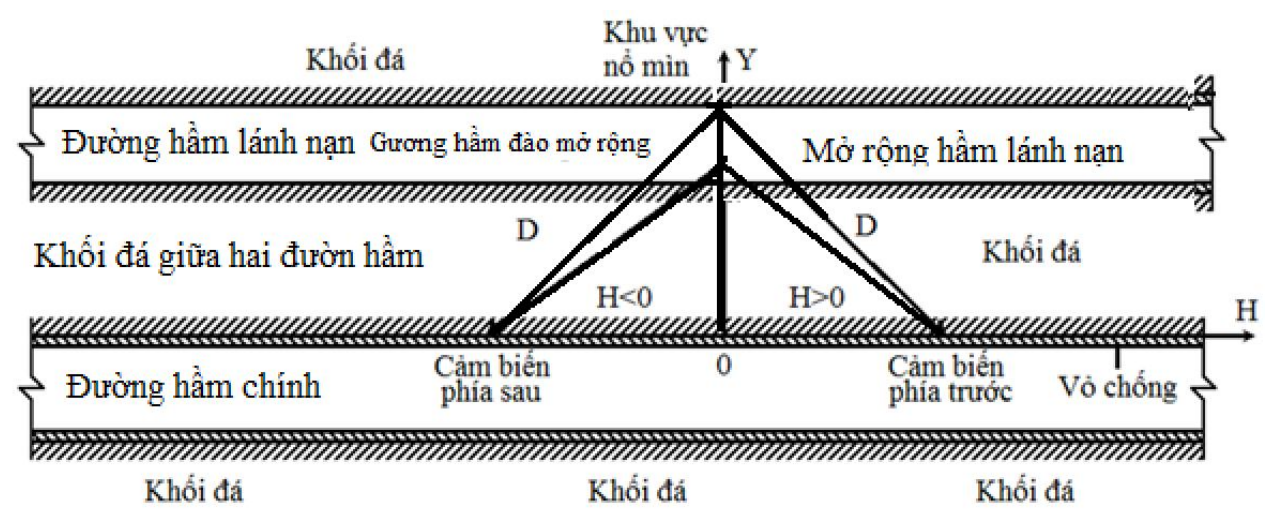

Hình 4. Sơ đồ nghiên cứu sự ảnh hương của chấn động nổ mìn tại gương hầm lánh nạn đến vỏ chống bê tông của đường hầm chính theo phương dọc trục hầm tại dự án hầm đường bộ qua đèo Hải Vân. 
a)
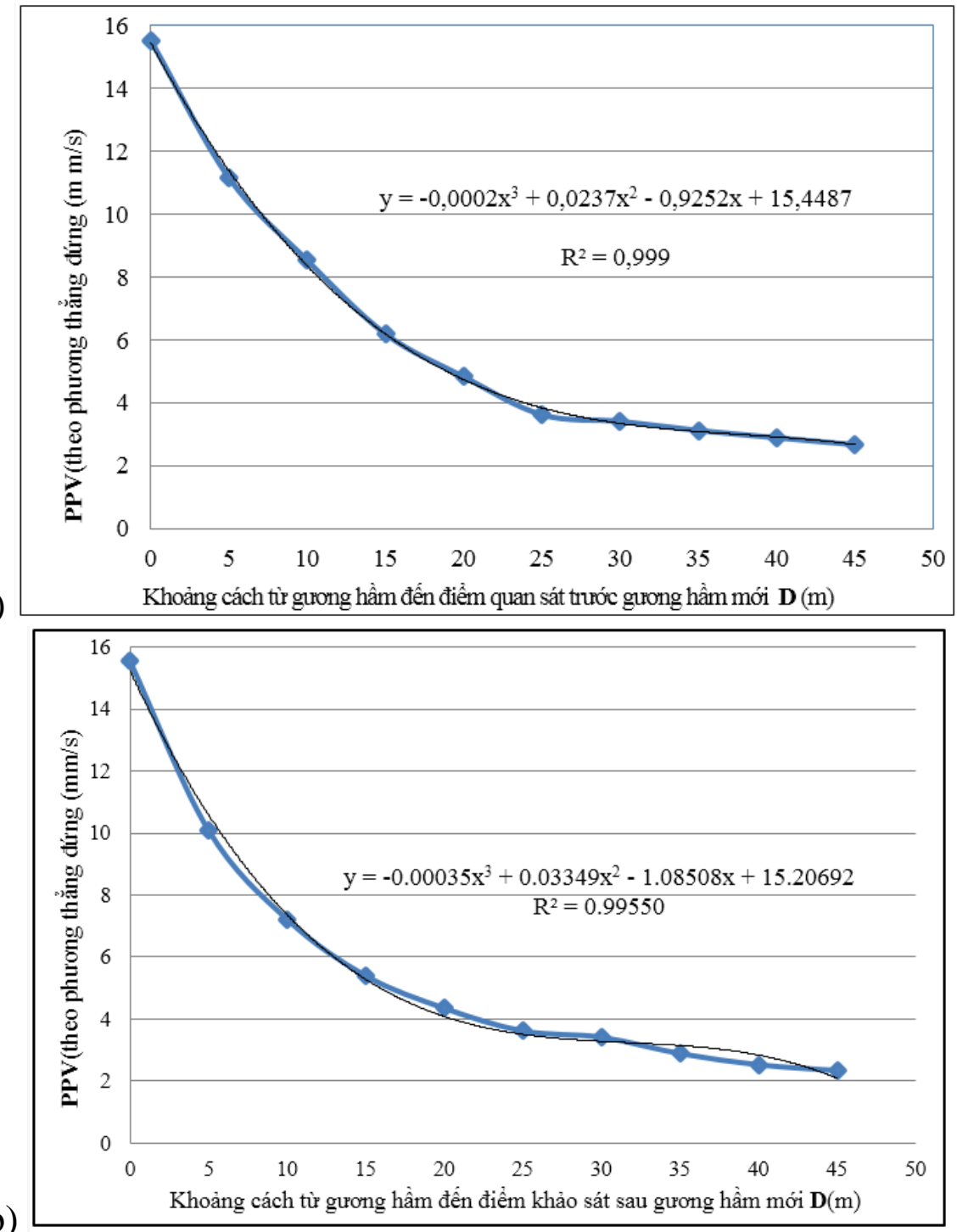

Hình 5. Môi quan giũa giá trị PPV và khoảng cách tính tù̀ gương

đưòng hầm đến các điểm khảo sát $(\mathrm{H})$. (Đường mầu xanh là đường gần đúng; đường màu đen là đồ thị phưong trình thu được)

Từ biểu đồ Hình 5 cho thấy giá trị vận tốc dao động đỉnh phần tử lớn nhất tỉ lệ nghịch với khoảng cách từ gương hầm đến vị trí quan sát dọc trục hầm chính, càng xa gương hầm lánh nạn, giá trị này càng giảm. Theo kết quả trên PPV là hàm bậc 3 của khoảng cách (D) với hệ số tương quan cao, lớn hơn 0,9 nên có độ tin cậy. Ngoài ra, các kết quả nghiên cứu tại dự án hầm Croix-Rousse đã được kiểm chứng bằng hai phương pháp số và phương pháp đo đạc thực nghiệm tại nên có độ tin cậy cao. Do vậy việc áp dụng kết quả tại dự án hầm đường bộ qua đèo Hải Vân hoàn toàn có sự tin cậy.

\section{Kết luận}

Các kết quả trong bài báo này cho phép rút ra một số kết luận được rút ra sau đây:

- Khoảng cách giữa hầm lánh nạn đang đào mở rộng và hầm chính là nhỏ đó đòi hỏi công tác khoan nổ mìn khi nổ mìn mở rộng cần được giám sát theo các tiêu chuẩn của Vlệt Nam như QCVN 022008 BCT.

-Việc áp dụng kết quả nghiên cứu trước cho hầm Croix-Rousse trong điều kiện tương tự cho hầm Hải Vân cho phép dự báo ảnh hưởng của chấn động nổ mìn hầm lánh nạn đến kết cấu chống của 
đường hầm chính. Kết quả là cơ sở quan trọng để điều chỉnh hộ chiếu khoan nổ mìn và biện pháp thi công hầm nhằm đảm bảo an toàn cho vỏ chống của hầm chính.

- Theo kết quả nghiên cứu, tại dự án hầm đường bộ qua đèo Hải Vân khi đào qua đá granite, giá trị PPV là hàm bậc 3 của khoảng cách (D) như các công thức (1) và (2), các công thức trên là kết quả áp dụng từ dự án hầm Croix-Rousse, Lyon, Pháp đã được xây dựng dựa trên hai phương pháp số và phương pháp đo đạc thực nghiệm hiện trường nên có độ tin cậy cao.

\section{Tài liệu tham khảo}

Dang V.K., Dias D., Do N.A., Vo T.H., (2018), Impact of Blasting at TunnelFace on an Existing Adjacent Tunnel. International Journal of GEOMATE, July, 2018 Vol.15, Issue 47, pp.2231 Geotec., Const. Mat. \& Env., ISSN: 21862982 (Print), 2186-2990 (Online), Japan.

Đặng Văn Kiên (2014). Khảo sát chấn độngnổ mìn khi đào hầm bằng phương pháp khoan nổ mìn ở khu vực thành phố bằng mô hình $2 \mathrm{D}, \mathrm{T}$ ạp chí Công nghiệp Mỏ, (6) , 2014, tr. 7-12.

Đặng Văn Kiên (2016). Nghiên cứu ảnh hưởng của chấn động nổ mìn đến kết cấu công trình ngầm lân cận bằng hai phương pháp đo đạc hiện trường và phương pháp số, Tạp chí Công nghiệp Mỏ, (3), tr. 17-21.

Đặng Văn Kiên, (2017). Nghiên cứu đánh giá ản hưởng của chấn động nổ mìn đến kết cấu cỏ chống của đường hầm chính tại dự án hầm Hải Vân khi tiến hành mở rộng hầm lánh nạn bằng phương pháp khoan nổ mìn, Tạp chí Công nghiệp Mỏ, (2), tr. 33-40.

Đặng Văn Kiên, (2018). Nghiên cúu ảnh hưởng của chấn động nổ mìn khi thi công đường hầm đến kết cấu công trình ngầm lân cận. Luận án Tiến Sỹ, Đại học Mỏ - Địa chất. Hà Nội, 179 trang.

Ichizuru ISHIMOTO and Tran Ngoc NGUYEN. Introduction of hai van pass tunnel construction project in vietnam. Project Management Unit No.85, Ministry of Transport, Vinh, Vietnam.

Keshavarz M., Dang V.K., Amini H.K., Pellet F.L., (2013). AE thresholds and compressive strength of different crystalline rocks subjected to static and dynamic loadings. 1st International Conference on Rock Dynamics and Applications. 06-08 June 2013, LausanneSwitzerland.

Olsson, M., Nie, S., Bergqvist, I., Ouchterlony, F. (2001): What causes cracks in rock blasting? In: Proc. EXPLO2001. Hunter valley, NSW, Australia, 191-196.

Pellet F. L., Dang V. K., Baumont C., Dusseux M., Huang G. J. Determination of dynamic rock strength to assess blasting efficiency, (2013). International conference on Rock Mechanics for Resources Energy, and Environment- Eurock 2013. 21-26 September 2013, Wroclaw, Poland.

Quy chuẩn kỹ thuật quốc gia QCVN 02:2008/ BCT. Về an toàn trong bảo quản, vận chuyển, sử dụng và tiêu hủy vật liệu nổ công nghiệp. National technical regulation on safety in the storage, transportation, use and disposal of industrial explosive materials. Hà Nội -2008.

Saharan, M. R. and Mitri, H. S. Numerical Procedure for Dynamic Simulation of Discrete Fractures Due to Blasting. Rock Mech. Rock Engng, 2008, 41 (5) : 641-670.

Van Kien DANG, Ali LIMAM, Didier SUBRIN, Emmanuel HUMBERT, (2013). Blast vibration induced during tunnel excavation in urban areas: Numerical simulation and measure results. Int. Conf. Franco - Vietnamienne CIGOS 2013 on Construction et Developpement Durable. 04-05 Avril 2013, Lyon,France.

Van Kien Dang, Trong Hung Vo, Ngoc Anh Do (2016). 2D/3D numerical simulation the effect of blast vibration on the existing tunnel during tunnel excavation by blasting method. Proceedings of the international conferences on earth sciences and sustainable geo-resources development (ESASGD 2016).

Võ Trọng Hùng, Đặng Văn Kiên, (2018). Nghiên cứu sử dụng chỉ số "RMR" để đánh giá chấn động do nổ mìn đào hầm đến kết cấu đường hầm lân cận thông qua vận tốc PPV, Tạp chí Công nghiệp Mỏ, (3), tr. 1-9. 